\title{
A Hybrid Retrieval Strategy for Case-based Reasoning Using Soft Likelihood Functions
}

\author{
Yameng Wang \\ Harbin Institute of Technology \\ Liguo Fei \\ Harbin Institute of Technology

\section{Yuqiang Feng} \\ Harbin Institute of Technology \\ Yanqing Wang \\ Harbin Institute of Technology \\ Luning Liu ( $\nabla$ liuluning@hit.edu.cn ) \\ Harbin Institute of Technology https://orcid.org/0000-0002-5539-5623
}

\section{Research Article}

Keywords: Case-based reasoning, Retrieval, Soft likelihood function, Ordered weighted average, Attitudinal character

Posted Date: June 28th, 2021

DOl: https://doi.org/10.21203/rs.3.rs-561791/v1

License: (c) (i) This work is licensed under a Creative Commons Attribution 4.0 International License. Read Full License

Version of Record: A version of this preprint was published at Soft Computing on January $21 \mathrm{st}, 2022$. See the published version at https://doi.org/10.1007/s00500-022-06733-5. 


\title{
A hybrid retrieval strategy for case-based reasoning using soft likelihood functions
}

\author{
Yameng Wang ${ }^{\mathrm{a}}$, Liguo Fei ${ }^{\mathrm{a}}$, Yuqiang Feng ${ }^{\mathrm{a}}$, Yanqing Wang ${ }^{\mathrm{a}}$, Luning Liu ${ }^{\mathrm{a}, *}$ \\ ${ }^{a}$ School of Management, Harbin Institute of Technology, Harbin 150001, China
}

\begin{abstract}
Case-based reasoning (CBR) is the retrieval of one or more similar cases from an existing case base for the problem to be solved according to the characteristics of the new problem. The core idea of CBR is that similar cases have similar solutions, so whether the CBR system can play a powerful advantage depends on the quality of case retrieval strategy. At present, the commonly used case retrieval algorithm is based on the mean operator method, which is very hard, and a certain local similarity is low will affect the overall result. In order to calculate the global similarity of cases from a new and softer point of view, this paper introduces the soft likelihood functions into case retrieval, combines the soft likelihood functions with KNN, and proposes a hybrid retrieval strategy. The core of the retrieval strategy is to define the global similarity through SLFs, aggregate the local similarity and characteristic similarity together, and also take the attitude characteristics of decision makers into consideration. Through simulation experiments on real data sets, the accuracy rate is more than $81 \%$, which verifies the effectiveness of the retrieval strategy.

Keywords: Case-based reasoning, Retrieval, Soft likelihood function, Ordered weighted average, Attitudinal character.
\end{abstract}

\section{Introduction}

The proposal of case-based reasoning (CBR) can be traced back to the late 1970s [1]. Roger et al. from Yale University in the United States proposed to represent knowledge by means of script, which is regarded as the beginning of CBR research. Since then, CBR has experienced from

*Corresponding author: Luning Liu, School of Management, Harbin Institute of Technology, Harbin 150001, China

Email address: liuluning@hit.edu.cn (Luning Liu) 
simple basic application research to theoretical perfection $[2,3,4,5,6]$. It originated in the fields of cognitive science (CS) and artificial intelligence (AI). Typically, the current problem or situation is referred to as the target case, and the problem or situation that has occurred is referred to as the source case or the historical case. Case-based reasoning refers to recalling previous successful cases which is referred to as the source case or historical case, by comparing the similarities and differences between the source case and target case, and then adjusting the target case solutions based on the successful cases to solve the current problem [7]. In particular, case-based reasoning plays a very important role in the field of application where there is no known standard, no known cycle, and no complete domain theory [8]. CBR can simplify knowledge acquisition, improve problem solving efficiency, improve solving quality, and accumulate knowledge. It provides a method which is quite similar to human solving problems [9].

At present, CBR has been widely used in AI, and it has become a new methodology of problem solving and learning [10]. With the gradual maturity of theories and methods, the applications of CBR have been extended to various fields, including medical treatment [11, 12, 13, 14, 15], planning $[16,17]$, assessment [18, 19], forecast [20, 21], game [22], recommendation system [23], management $20 \quad[24]$ and so on $[25,26]$.

The core idea of CBR is that similar cases have similar solutions. Plenty of scholars developed different case-based reasoning models with the intention of providing a better understanding of casebased reasoning process. One of the representative models is the CBR model introduced by Aamodt and Plaza [27], in which they propose a process for solving a new problem. Before reasoning, we need to choose the appropriate method to build the case base [28]. For a problem to be solved, one or more similar cases are retrieved from the existing case base according to the features of the new problem. Solutions to cases retrieved are employed to generate solutions to the new problem and the solutions will be tested, modified, and evaluated to determine their effectiveness. Solutions that satisfy the user are learned and added to the case bases. The model of the CBR cycle is illustrated in Fig. 1, which is called the 4- $R$ lifecycle model.

From the model of CBR cycle, the CBR reasoning process is mainly divided into four stages: retrieval $(R-1)$, reuse $(R-2)$, revise $(R-3)$ and retain $(R-4)[29]$.

- $R$-1: RETRIEVE information from the source case base and select potentially available source cases. 


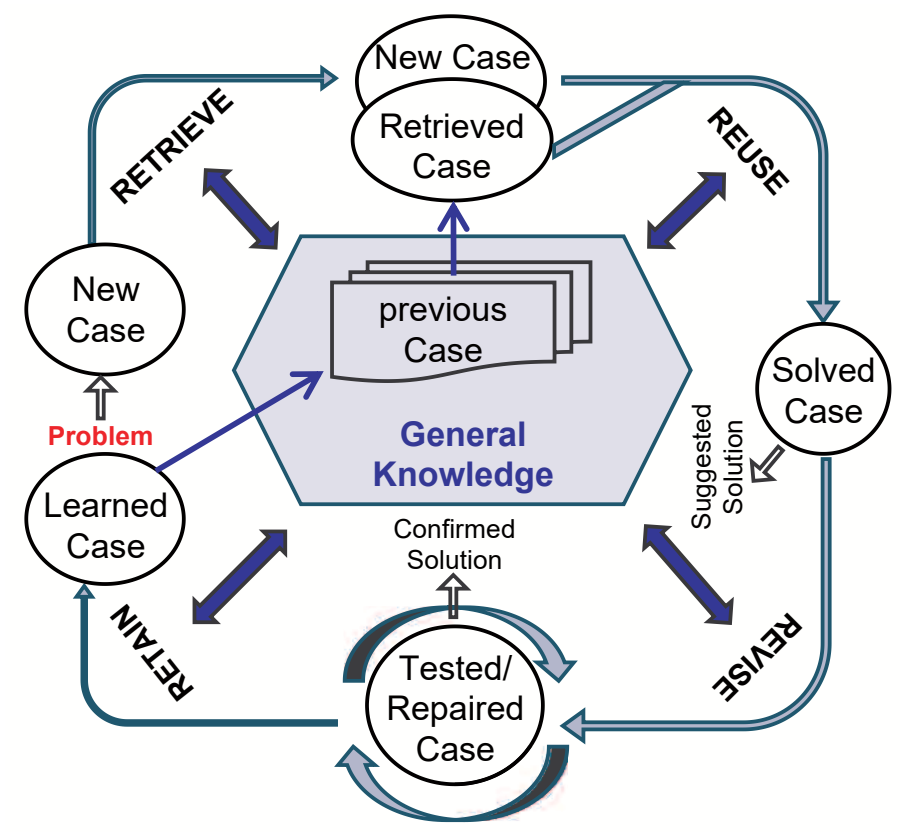

Figure 1: The 4-R lifecycle model of CBR from Aamodt and Plaza [27].

- $R$-2: REUSE the solutions of the retrieved source cases in new problems or cases.

- $R$-3: REVISE the proposed solution.

- $R$-4: RETAIN the solution in favor of subsequent reasoning in the problem.

The 4- $R$ cycle model is summarized as: analyze the features of existing problems, retrieve one or more similar cases, try to reuse cases, and retain new cases in case base according to their importance after the solution is revised and applied.

From the 4- $R$ cycle model we can get the fact that whether CBR system could play a strong advantage depends on the quality of case retrieval strategy [30]. The retrieval method directly affects the retrieval speed and accuracy rate [31], and whether the retrieval strategy is reasonable or not directly affects the realization effect of the whole case system. So case retrieval is the key to problem solving. In the aspect of retrieval strategy, there are knowledge guidance strategy [32], template retrieval strategy [33] and nearest neighbor strategy [34, 35].

From the research status of case retrieval at home and abroad [36], the K-nearest neighbor (KNN) retrieval strategy [34] is widely used at present [37]. It calculates the similarity between 
the target case to be solved and the source case in the case base according to the attribute weight as the basis of case reuse [39]. In the calculation of similarity, the weight distribution will have a significant influence on the calculation results and the quality of the solution. Attributes that generally play a major role are assigned greater weight; Conversely, less weight is given. KNN generally uses the average weight method. Although it is simple and easy to operate, it is sensitive this problem usually depends on the reasonable allocation of the weight of characteristic attributes, so the allocation of weight has become an important research direction.

On the one hand, although the similarity-based retrieval method has been successfully applied to $\mathrm{CBR}$ and received extensive attention, it is not completely consistent with the actual reasoning certain term. On the other hand, the CBR system was developed for use by decision makers (DMs), which inevitably reflects DMs' personal attitude in different situations. However, the attitude characteristics of DMs are often ignored in similarity calculation, which is unreasonable. Therefore, it is necessary to further study the mechanism of optimal weight allocation in order to improve the quality of problem solving.

Based on the above analysis, inspired by the soft likelihood functions (SLFs) introduced by Yager et al. [40], a new case retrieval algorithm using SLFs based ordered weighted average (OWA) (abbreviated as CBR-SLFs) is proposed in this study, which provides a new perspective to retrieve similar cases. The basic idea of case retrieval by the proposed method is as follows: Firstly, calculate the local similarity between different attributes of the target case and the source case; Then, the CBR-SLFs algorithm proposed in this paper is used to calculate the overall similarity, and some potential available source cases with high similarity are obtained; Finally, the source case solution that is closest to the target case is obtained through KNN, and reuse it. This strategy is developed as a flexible computation of likelihood functions of global similarity calculation, and has the advantage algorithm is developed introducing an attitudinal characteristic to reflect the subjective preference of decision makers, which allows for more flexible choices based on different types of decision makers.

The rest of this article is organized as follows: Section 2 briefly introduces likelihood function in case retrieval, some basic calculations of the OWA aggregation operator and local similarity 
measurement method for heterogeneous information. Section 3 introduces the application of soft likelihood function in case retrieval, then takes feature similarity into consideration and gives some examples. Section 4 provides some simulation experiments on benchmark data sets. Finally, section 5 summarizes this paper and puts forward the future research direction.

\section{Preliminaries}

This section first presents the likelihood functions in case retrieval and OWA aggregation, then introduces local similarity measurement methods for case information.

\subsection{Using likelihood functions in case retrieval}

In a CBR system, existing knowledge or experience needs to be represented as a case library that typically contains multiple cases. Each case is generally composed of two parts, the description of the problem and the corresponding solution, for the convenience of description, the symbol is given below.

$$
C_{i}=\left\{D_{i}, S_{i}\right\}, i=1,2, \ldots, n
$$

$C=\left\{C_{1}, C_{2}, \ldots, C_{n}\right\}$ is n historical cases in the case base, $C_{i}$ represents the $i$ th case $(i \in\{1,2, \ldots, n\})$ including problem description $D_{i}$ and corresponding solution $S_{i} . \mathcal{C} *$ is the target case, and the problem description for the target case is represented as $\mathcal{D} *$. Suppose $S I M_{i}$ represents the similarity between $\mathrm{Ci}$ and the target case. $\operatorname{Sim}_{j}\left(\mathcal{D} *, D_{i}\right)$ represents the similarity of the problem description $\mathcal{D} *$ of the target case and the problem description $D_{i}$ of the historical case $C_{i}$ about the characteristic attribute $j$.

In case reasoning, our goal is to find some order of historical cases in the case base, that is, the similarity between historical cases and target cases, so as to support the selection of source cases with the highest similarity as candidate cases for further revision and use. In other words, the more similar the historical case is, the more willing we are to reuse the case. One way to calculate the similarity of a case is to take the product of the local similarity of different attributes.

$$
S I M_{i}=\prod_{j=1}^{q} \operatorname{sim}_{i j}
$$


We can see that each additional feature can only reduce the probability that the case $C_{i}$ is the best candidate case. If any $\operatorname{sim}_{i j}=0$ for $j=1 \ldots q$, then $S I M_{i}=0$. More generally, we observe that for any case $C_{i}$, as long as there is a low local similarity value, the overall similarity of the case $C_{i}$ will be greatly reduced. This is a kind of logical anding for a given $C_{i}$. The expression of this possibility is too strong, because it requires the premise that all the local similarity of $C_{i}$ is consistent and high, so that we can think of this suspect's historical case as similar. Therefore, this paper will consider the use of OWA aggregation operator to determine the candidate case similarity of the softer formula. In the following text, we set $\lambda_{i}$ as the index function and $\lambda_{i}(k)$ as the $k$ th probability index of great compatibility of $C_{i}$. Here $\operatorname{sim}_{i \lambda_{i}(k)}$ is the $k$ th largest local similarity of the case $C_{i}$. We let:

$$
\operatorname{Prod}_{i}(j)=\prod_{k=1}^{j} \operatorname{sim}_{i \lambda_{i}(k)}
$$

Here $\operatorname{Prod}_{i}(j)$ is the product of the $\mathrm{j}$ largest probabilities. We note that $\operatorname{Prod}_{i}(j)$ is monotonically decreasing as a function of $\mathbf{j}$, that is if $j_{1}<j_{2}$, then $\operatorname{Prod}_{i}\left(j_{1}\right) \geq \operatorname{Prod}_{i}\left(j_{2}\right)$. Also we can easily observe that $\operatorname{Prod}_{i}(j) \in[0,1]$ since each $\operatorname{sim}_{i \lambda_{i}(k)} \in[0,1]$.We observe that the likelihood function can now be expressed as $S I M_{i}=\operatorname{Prod}_{i}(q)$.

\subsection{Ordered weight averaging aggregation}

Below, we will consider using OWA aggregation operator to provide a class of soft likelihood functions based on $\operatorname{Prod}_{i}(j)$. In order to do this, we need to briefly describe the OWA aggregation

Ordered weight averaging aggregation was first proposed by Yager [42]. An OWA aggregator operator of $\mathrm{n}$ dimension is a mapping: $R^{n} \rightarrow R$. $O W A_{w}\left(a_{1}, a_{2}, \ldots, a_{i}, \ldots, a_{n}\right)=\sum_{j=1}^{n} w_{j} a_{\lambda(j)}$, where $W=\left(w_{1}, w_{2}, \ldots, w_{n}\right)^{T}$ is the weighted vector associated with the function OWA with $w_{j} \epsilon[0,1]$ and $\sum_{j} w_{j}=1(j \epsilon\{1,2, \ldots, n\}) ; a_{\lambda(j)}$ is the $j t h$ largest element in $a_{1}, a_{2}, \ldots, a_{n}$ in order from largest to smallest. Then we called function OWA as ordered weight averaging operator, which is also called OWA operator.

The characteristic of OWA operator is to rearrange the given data $\left(a_{1}, a_{2}, \ldots, a_{i}, \ldots, a_{n}\right)$ into $\left(a_{\lambda(1)}, a_{\lambda(2)}, \ldots, a_{\lambda(i)}, \ldots, a_{\lambda(n)}\right)$ in order from large to small, and aggregate $\left(a_{\lambda(1)}, a_{\lambda(2)}, \ldots, a_{\lambda(i)}\right.$, $\left.\ldots, a_{\lambda(n)}\right)$ by the given weight vector. Furthermore, element $a_{i}$ has nothing to do with weight $w_{j}$, 
and weight $w_{j}$ is only related to the $j t h$ position in the assembly process, so the weighted vector $W$ is also called the position weighted vector.

Let's notice some special operators[42]:

1. $W^{*}=(1,0, \ldots, 0)$, the OWA operator is reduced to the max operator, $O W A\left(a_{1}, \ldots, a_{n}\right)=$ $a_{\lambda(1)}=\max _{i}\left(a_{i}\right)$.

2. $W_{*}=(0,0, \ldots, 1)$, the OWA operator is reduced to the min operator, $O W A\left(a_{1}, \ldots, a_{n}\right)=$ $a_{\lambda(n)}=\min _{i}\left(a_{i}\right)$.

3. $W_{n}=\left(\frac{1}{n}, \frac{1}{n}, \ldots, \frac{1}{n}\right)$, the OWA operator is reduced to a simple arithmetic average operator, $O W A\left(a_{1}, \ldots, a_{n}\right)=\frac{1}{n} \sum_{i=1}^{n} a_{i}$.

4. $W_{n-2}=\left(0, \frac{1}{n-2}, \frac{1}{n-2}, \ldots, \frac{1}{n-2}, 0\right)$, the OWA operator is reduced to an arithmetic average operator that removes the extremum, $O W A\left(a_{1}, \ldots, a_{n}\right)=\frac{1}{n-2}\left(\sum_{i=1}^{n} a_{i}-\max _{i}\left(a_{i}\right)-\min _{i}\left(a_{i}\right)\right)$

5. $W_{k}=(0, \ldots, 1, \ldots, 0), O W A\left(a_{1}, \ldots, a_{n}\right)=a_{\lambda(k)}$.

We can discover that the more weight is assigned to $w_{j}$ near the top of $W$ (the $w_{j}$ with a smaller index), the aggregate value is larger; while the more weight is assigned to $w_{j}$ near the bottom of $W$ (the $w_{j}$ with a larger index), the aggregate value is smaller. Weighted vector $W$ that can reflect the tendency of the DMs to be optimistic or pessimistic and it determines how OWA is aggregated. Attitudinal character is defined as[43]:

$$
A C(W)=\sum_{j=1}^{n} \frac{n-j}{n-1} w_{j}
$$

We can find out that $A C(W) \in[0,1]$ and $A C\left(W^{*}\right)=1, A C\left(W_{*}\right)=0, A C\left(W_{n}\right)=0.5, A C\left(W_{n-2}\right)=$ $0.5, A C\left(W_{k}\right)=\frac{n-k}{n-1}$. The value of attitudinal character $A C(W)$ determines the degree of optimism. In other words, the larger the attitudinal character is, the more optimistic it is and the higher the aggregated value is.

We use a method to get OWA weights- $w_{j}$. Assume function $\mathrm{f:}[0,1] \rightarrow[0,1]$ is monotonic; when $x>y, f(x)>f(y) ; f(0)=0$ and $f(1)=1$. We obtain:

$$
w_{j}=f\left(\frac{j}{n}\right)-f\left(\frac{j-1}{n}\right)
$$

$w_{j} \in[0,1]$ and $\sum_{j=1}^{n} w_{j}=1 ; w_{j}$ has all the properties of the OWA weights [43].

We call this method of obtaining OWA weights the function method, where $w_{j}$ and the associated attitudinal character depends not only on the function itself, but also on the cardinality $\mathrm{n}$. 
Attitudinal character is defined as [43]:

$$
\operatorname{Opt}(f)=\int_{0}^{1} f(x) d x
$$

When n gets really big, $\operatorname{Opt}(f)$ is really just $A C(W)$.

We can find $f(x)=x^{m}$ for $m \geq 0$, and for this function,

$$
\alpha=\int_{0}^{1} x^{m} d x=\left.\frac{x^{m+1}}{m+1}\right|_{0} ^{1}=\frac{1}{m+1}
$$

We have $m=\frac{1-\alpha}{\alpha}$, and $\alpha \in[0,1]$. We can see the larger $\alpha$ is, the more optimistic the attitude of users is. $m=1$ when $\alpha=0.5 ; m=0$ when $\alpha=1 ; m \rightarrow \infty$ when $\alpha \rightarrow 0$.

Using the above functional form, the OWA weights is:

$$
w_{j}=f\left(\frac{j}{n}\right)-f\left(\frac{j-1}{n}\right)=\left(\frac{j}{n}\right)^{m}-\left(\frac{j-1}{n}\right)^{m}
$$

Then for a given $\alpha$, we can obtain:

$$
w_{j}=\left(\frac{j}{n}\right)^{\frac{1-\alpha}{\alpha}}-\left(\frac{j-1}{n}\right)^{\frac{1-\alpha}{\alpha}}
$$

Then we shall consider softer formulations for determining candidate similarity by using the OWA aggregation operator.

\subsection{Local similarity measurement methods for case information}

CBR is very similar to the way humans solve problems. When a new problem is encountered, it uses retrieval method to retrieve and select potentially available source cases from the source case base [44]. CBR can not only give full play to the advantage of the immediacy of computer processing information, but also improve the scientific nature and effectiveness of decision making [45]. In the case-based reasoning system, whether all the follow-up work can play its due role largely depends on the quality of the cases retrieved, so case retrieval is very critical.

The information or data in a case-based reasoning system is usually heterogeneous, and heterogeneity indicates a difference in the type and nature of information or data [46]. The processing of heterogeneous information is a key point in the decision-making process [47, 48]. As case events are usually characterized by risk, complexity and uncertainty [49], plus the imprecision of the environment, decision information is often not always expressed as accurate numbers, including 
Boolean values, interval numbers and fuzzy numbers. In addition, because of the fuzziness of human thinking, it is sometimes difficult to express the decision information with quantitative values in the decision-making process, and qualitative language information is also used to evaluate the attributes [50].

Suppose $\operatorname{Sim}_{j}\left(\mathcal{D} *, D_{i}\right)$ represents the similarity between the target case $\mathcal{D} *$ and the historical case $D_{i}$ about the characteristic attribute $j$. Heterogeneous decision information contains many types of attribute information such as numerical features, Boolean features, symbolic features with orders, symbolic features without orders, string features, fuzzy features, interval features, and its similarity is calculated as follows [51].

- for numerical features, the similarity between $\mathcal{D} *$ and $D_{i}$ can be obtained as

$$
\operatorname{Sim}_{j}\left(\mathcal{D} *, D_{i}\right)=1-\frac{\left|\mathcal{D} *-D_{i}\right|}{\max }
$$

- for Boolean features, the similarity between $\mathcal{D} *$ and $D_{i}$ can be obtained as

$$
\operatorname{Sim}_{j}\left(\mathcal{D} *, D_{i}\right)= \begin{cases}0 & \mathcal{D} * \neq D_{i} \\ 1 & \mathcal{D} *=D_{i}\end{cases}
$$

- for symbolic features with orders, the similarity between $\mathcal{D} *$ and $D_{i}$ can be obtained as

$$
\operatorname{Sim}_{j}\left(\mathcal{D} *, D_{i}\right)=1-\frac{\left|\mathcal{D} *-D_{i}\right|}{g}
$$

where $\mathrm{g}$ is the number of value levels.

- for symbolic features without orders, the similarity between $\mathcal{D} *$ and $D_{i}$ can be obtained as

$$
\operatorname{Sim}_{j}\left(\mathcal{D} *, D_{i}\right)=\frac{\operatorname{num}\left(\mathcal{D} * \wedge D_{i}\right)}{n u m\left(\mathcal{D} * \vee D_{i}\right)}
$$

- for string features, the similarity between $\mathcal{D} *$ and $D_{i}$ can be obtained as

$$
\operatorname{Sim}_{j}\left(\mathcal{D} *, D_{i}\right)=\frac{t \times l}{\max \left(\operatorname{len}(\mathcal{D} *), \operatorname{len}\left(D_{i}\right)\right)}
$$

where $t$ is the matching number, $l$ is the matching length and len is the string length.

- for fuzzy features, the similarity between $\mathcal{D} *$ and $D_{i}$ can be obtained as

$$
\begin{array}{r}
\operatorname{Sim}_{j}\left(\mathcal{D} *, D_{i}\right)=1-\left\{\left(n_{i}-n_{i}^{\prime}\right)^{2}+\frac{1}{9}\left[\left(m_{i}-m_{i}^{\prime}\right)^{2}+\left(r_{i}-r_{i}^{\prime}\right)^{2}-\left(m_{i}-m_{i}^{\prime}\right)\left(r_{i}-r_{i}^{\prime}\right)\right]\right. \\
\left.-\frac{1}{2}\left(n_{i}-n_{i}^{\prime}\right)\left[\left(m_{i}-m_{i}^{\prime}\right)-\left(r_{i}-r_{i}^{\prime}\right)\right]\right\}^{\frac{1}{2}}
\end{array}
$$

$\mathcal{D} *, D_{i}$ are triangular fuzzy number, $\mathcal{D} *=\left(n_{i}, m_{i}, r_{i}\right), D_{i}=\left(n_{i}^{\prime}, m_{i}^{\prime}, r_{i}^{\prime}\right)$ 
- for interval features, the similarity between $\mathcal{D} *$ and $D_{i}$ can be obtained as

$$
\operatorname{Sim}_{j}\left(\mathcal{D} *, D_{i}\right)=\frac{\operatorname{len}\left(\mathcal{D} * \bigcap D_{i}\right)}{\operatorname{len}(\mathcal{D} *)+\operatorname{len}\left(D_{i}\right)-\operatorname{len}\left(\mathcal{D} * \bigcap D_{i}\right)}
$$

where len is the interval length, $\mathcal{D} * \bigcap D_{i}$ is the overlapping interval.

\section{Case retrieval strategy}

In this section, we first propose a new global similarity calculation method based soft likelihood function that integrates the similarity of each attribute, and then, considering the feature similarity, we give a SLFs case retrieval algorithm combining the feature similarity. Our retrieval strategy is to combine case retrieval algorithm based on soft likelihood functions with KNN, so as to improve the performance of case retrieval.

\subsection{Case retrieval method based on soft likelihood functions}

In the previous section, we have obtained the local attribute similarity between the target case and the historical case under a variety of heterogeneous information environments. The global similarity is then calculated to retrieve the historical cases that are most similar to the target cases from the case base. We apply the soft likelihood functions based on OWA to the case retrieval process, and propose a new global similarity calculation method to improve the previous case retrieval strategy.

Let's consider using soft likelihood functions based OWA as a retrieval strategy for case-based reasoning. For each source case $C_{i}$ that we denote global similarity as $S I M_{i, W}$, we use the weighting vector $W$ and the $\operatorname{Prod}_{i}(j)$ to calculate it. Here $W=\left\{w_{1}, \ldots, w_{q}\right\}, w_{j} \in[0,1], \sum_{j=1}^{n} w_{j}=1$, and characterizes how we define this softer aggregation function. We define

$$
\operatorname{SIM}_{i, W}=\sum_{j=1}^{q} w_{j} \operatorname{Prod}_{i}(j)
$$

where as we have already indicated $\operatorname{Prod}_{i}(j)=\prod_{k=1}^{j} \operatorname{sim}_{i \lambda_{i}(k)}$. Here $\lambda_{i}$ is an index function so that $\lambda_{i}(k)$ is an index of the local similarity of attribute with the $k$ th largest probability of compatibility of case $C_{i}$.

For each $C_{i}, \operatorname{Prod}_{i}(j)=\operatorname{Prod}_{i}(j-1) \operatorname{sim}_{i \lambda_{i}(k)}$, as $\operatorname{sim}_{i \lambda_{i}(k)} \leq 1$, so $\operatorname{Prod}_{i}(j)$ is $\operatorname{monotonic}$ decreasing in $\mathrm{j}$, and $\operatorname{Prod}_{i}(j) \geq \operatorname{Prod}_{i}\left(j^{\prime}\right)$ for $j<j^{\prime}$. Therefor, the $\operatorname{Prod}_{i}(j)$ for $j=1 \ldots q$ using the 
weighting vector $W$ based on the OWA aggregation is

$$
\operatorname{SIM}_{i, W}=\sum_{j=1}^{q} w_{j} \operatorname{Prod}_{i}(j)=O W A_{W}\left\{\operatorname{Prod}_{i}(1), \ldots, \operatorname{Prod}_{i}(q)\right\}
$$

The actual form of the soft likelihood functions is determined by the weighting vector $W$ which is only related to the location. For some of the special weighting vector,

(1): $W^{*}=\left\{w_{1}=1, w_{j}=0 \mid j=2, \ldots, q\right\}$, we see easily that in this case

$$
\operatorname{SIM}_{i, W^{*}}=\operatorname{Prod}_{i}(1)=\operatorname{sim}_{i \lambda_{i}(1)}
$$

This is a kind of simple average of the $\operatorname{Prod}_{i}(j)$.

(4): $W_{n}=\left\{w_{1}=0, w_{j}=0, w_{j}=\frac{1}{q-2} \mid j=2, \ldots, q-1\right\}$, we see easily that in this case

$$
\begin{aligned}
\operatorname{SIM}_{i, W_{n}} & =\frac{1}{q-2}\left(\sum_{j=1}^{q} \operatorname{Prod}_{i}(j)-\operatorname{Prod}_{i}(1)-\operatorname{Prod}_{i}(q)\right) \\
& =\frac{1}{q-2}\left(\sum_{j=1}^{q}\left(\prod_{k=1}^{j} \operatorname{sim}_{i \lambda_{i}(k)}\right)-\operatorname{sim}_{i \lambda_{i}(1)}-\prod_{j=1}^{q} \operatorname{sim}_{i j}\right)
\end{aligned}
$$

This is a kind of arithmetic average of the $\operatorname{Prod}_{i}(j)$ which removes the extremum.

If we are more optimistic to the likelihood, more of the allocated weight is related to the $w_{j}$ that has more smaller indices; but if we are more pessimistic to the likelihood, more of the allocated weight is related to the $w_{j}$ that has more larger indices. Due to $S I M_{i, W}$ is depending on the $W$, so 

the weighting vector $W$. If the user is more optimistic, then the $\alpha$ is closer to 1 and the value of $S I M_{i, W_{N}}$ is larger; while the user is more pessimistic, then the $\alpha$ is closer to 0 and the value of $S I M_{i, W_{N}}$ is smaller.

As we discussed earlier $w_{j}=f\left(\frac{j}{q}\right)-f\left(\frac{j-1}{q}\right)$ and $f(x)=x^{m}$. In addition, we use $m=\frac{1-\alpha}{\alpha}$ to show the desired degree of optimum $\alpha$. As a result, we can express users' attitude by a softer likelihood function which is more in line with the reality. We can get:

$$
\left.S I M_{i, \alpha}=\sum_{j=1}^{q}\left[\left(\frac{j}{q}\right)^{\frac{1-\alpha}{\alpha}}-\left(\frac{j-1}{q}\right)^{\frac{1-\alpha}{\alpha}}\right] \prod_{k=1}^{j} \operatorname{sim}_{i \lambda_{i}(k)}\right)
$$

Because of the physiological and cognitive limitations of the DMs, he is bounded rational in

Then the index function $\lambda_{i}(k)$ is such that $\lambda_{i}(1)=4, \lambda_{i}(2)=3, \lambda_{i}(3)=6, \lambda_{i}(4)=1, \lambda_{i}(5)=$ $5, \lambda_{i}(6)=2$ to order the probabilities. From these values we can calculate $\operatorname{Prod}_{i}(j)=\prod_{k=1}^{j} \operatorname{sim}_{i \lambda_{i}(k)}$ and these results is in Table 1.

The value of $\alpha$ is different for different users and we can calculate some typical $S I M_{i, \alpha}$. For ${ }_{230} q=6, w_{j}=\left(\frac{j}{6}\right)^{\frac{1-\alpha}{\alpha}}-\left(\frac{j-1}{6}\right)^{\frac{1-\alpha}{\alpha}}$ and $S I M_{i, \alpha}=\sum_{j=1}^{6} w_{j} \operatorname{Prod}_{i}(j)$. 
Table 1: Probability products

\begin{tabular}{ll}
\hline Ordered probability & $\operatorname{Prod}_{i}(j)$ \\
\hline $\operatorname{sim}_{i \lambda_{i}(1)}=\operatorname{sim}_{i 4}=1$ & $\operatorname{Prod}_{i}(1)=1$ \\
$\operatorname{sim}_{i \lambda_{i}(2)}=\operatorname{sim}_{i 3}=0.9$ & $\operatorname{Prod}_{i}(2)=1 \times 0.9=0.9$ \\
$\operatorname{sim}_{i \lambda_{i}(3)}=\operatorname{sim}_{i 6}=0.8$ & $\operatorname{Prod}_{i}(3)=0.9 \times 0.8=0.72$ \\
$\operatorname{sim}_{i \lambda_{i}(4)}=\operatorname{sim}_{i 1}=0.7$ & $\operatorname{Prod}_{i}(4)=0.72 \times 0.7=0.504$ \\
$\operatorname{sim}_{i \lambda_{i}(5)}=\operatorname{sim}_{i 5}=0.5$ & $\operatorname{Prod}_{i}(5)=0.504 \times 0.5=0.252$ \\
$\operatorname{sim}_{i \lambda_{i}(6)}=\operatorname{sim}_{i 2}=0.4$ & $\operatorname{Prod}_{i}(6)=0.252 \times 0.4=0.1008$ \\
\hline
\end{tabular}

(1) $\alpha=0.8$ : This is a very optimistic attitudinal character. $m=\frac{1-\alpha}{\alpha}=0.25$ and $w_{j}=$ $\left(\frac{j}{6}\right)^{0.25}-\left(\frac{j-1}{6}\right)^{0.25}$. The results are given in Table 2 .

Table 2: The numerical example of $\alpha=0.8$

\begin{tabular}{llllll}
\hline & $\left(\frac{j}{6}\right)^{0.25}$ & $\left(\frac{j-1}{6}\right)^{0.25}$ & $w_{j}$ & $\operatorname{Prod}_{i}(j)$ & $w_{j} \operatorname{Prod}_{i}(j)$ \\
\hline$j=1$ & 0.6389 & 0 & 0.6389 & 1 & 0.6389 \\
$j=2$ & 0.7598 & 0.6389 & 0.1209 & 0.9 & 0.1088 \\
$j=3$ & 0.8409 & 0.7598 & 0.0811 & 0.72 & 0.0584 \\
$j=4$ & 0.9036 & 0.8409 & 0.0627 & 0.504 & 0.0316 \\
$j=5$ & 0.9554 & 0.9036 & 0.0518 & 0.252 & 0.0131 \\
$j=6$ & 1 & 0.9554 & 0.0446 & 0.1008 & 0.0045 \\
& & & $\sum_{j} w_{j}=1$ & & $\sum_{j} w_{j} \operatorname{Prod}_{i}(j)=0.8553$ \\
\hline
\end{tabular}

So $S I M_{i, \alpha}=0.8553$ when $\alpha=0.8$.

(2) $\alpha=0.5$ : This is a very neutral attitudinal character. $m=\frac{1-\alpha}{\alpha}=1$ and $w_{j}=\left(\frac{j}{6}\right)-\left(\frac{j-1}{6}\right)=$

$\frac{1}{6}$. We can get: $\operatorname{SIM}_{i, \alpha}=\frac{1}{6} \sum_{j=1}^{6} \operatorname{Prod}_{i}(j)=\frac{1}{6}(1+0.9+0.72+0.504+0.252+0.1008)=0.579$. So $S I M_{i, \alpha}=0.579$ when $\alpha=0.5$.

(3) $\alpha=0.2$ : This is a very pessimistic attitudinal character. $m=\frac{1-\alpha}{\alpha}=4$ and $w_{j}=\left(\frac{j}{6}\right)^{4}-$ $\left(\frac{j-1}{6}\right)^{4}$. The results are given in Table 3. So $S I M_{i, \alpha}=0.2393$ when $\alpha=0.8$.

We can find from these examples that as $\alpha$ increases, so does $S I M_{i, \alpha}$. We see that the order of $C_{i}$ basically depends on the order of $\operatorname{sim}_{i j}$ via the indexing function $\lambda_{i}(k)$. Here for a given case $C_{i}$, the smaller the probability of a piece of attribute the lower it is in the ordering. 
Table 3: The numerical example of $\alpha=0.2$

\begin{tabular}{llllll}
\hline & $\left(\frac{j}{6}\right)^{4}$ & $\left(\frac{j-1}{6}\right)^{4}$ & $w_{j}$ & $\operatorname{Prod}_{i}(j)$ & $w_{j} \operatorname{Prod}_{i}(j)$ \\
\hline$j=1$ & 0.0008 & 0 & 0.0008 & 1 & 0.0008 \\
$j=2$ & 0.0123 & 0.0008 & 0.0116 & 0.9 & 0.0104 \\
$j=3$ & 0.0625 & 0.0123 & 0.0502 & 0.72 & 0.0361 \\
$j=4$ & 0.1975 & 0.0625 & 0.1350 & 0.504 & 0.0681 \\
$j=5$ & 0.4823 & 0.1975 & 0.2847 & 0.252 & 0.0718 \\
$j=6$ & 1 & 0.4823 & 0.5177 & 0.1008 & 0.0522 \\
& & & $\sum_{j} w_{j}=1$ & & $\sum_{j} w_{j} \operatorname{Prod}_{i}(j)=0.2393$ \\
\hline
\end{tabular}

\subsection{SLFs case retrieval algorithm combined with feature similarity}

When CBR is carried out, the attributes of the target case and the attributes of the source case in the case base are not necessarily the same [55], that is, we need to consider the feature similarity [56]. To solve the global similarity, both local similarity and feature similarity should be taken into consideration. In case retrieval, feature similarity is represented by different reliability of each attribute [57]. Therefore, the reliability of each attribute should be taken into consideration in the case retrieval algorithm of SLFs.

The reliability of each attribute is represented by $R_{i j}=\left\{r_{i 1}, r_{i 2}, \ldots r_{i q}\right\}, R_{i j} \epsilon[0,1]$, and $r_{i j}(j \epsilon 1,2$, $\ldots, q)$ represents the reliability of attribute $\mathrm{j}$ of the historical case i. In a case search, the reliability of each attribute does not change. So in this case, the value of $r_{i j}$ depends only on j, not on i. Next we give a description of SLFs case retrieval algorithm considering reliability [40].

First of all, let's calculate the total reliability as $R_{i}=\sum_{j=1}^{q} R_{i j}$, and then we use this to calculate the associated normalized reliability $r_{i j}=\frac{R i j}{R_{i}}$. Obviously, $\sum_{j=1}^{q} r_{i j}=1$.

We need to consider the products of the probability and the normalized reliability associated with target case $C_{i}$ and then calculate the soft likelihood in the face of reliability associated with each $\operatorname{sim}_{i j}$. We define an index function $\sigma_{i}$ and $\sigma_{i}(k)$ is the index of the $k t h$ largest of these products. So $\operatorname{sim}_{i \sigma_{i}(k)} \times r_{i \sigma_{i}(k)}$ is the $k t h$ largest of the $\operatorname{sim} \times r$ where $\operatorname{sim}_{i \sigma_{i}(k)}$ is the probability corresponding to the $k t h$ largest of the $\operatorname{sim} \times r$ products associated with $C_{i}$ and $r_{i \sigma_{i}(k)}$ is its associated reliability.

For a given $C_{i}$, the order of the local similarity is based on the product of the probability of compatibility of the local similarity of each attribute and the reliability of each attribute. The 
smaller this product the lower the piece of local similarity in the ordering. Either a small probability of compatibility or a small reliability can lead to a lower ordering. If all the attribute have the same reliability, then the index $\sigma_{i}(k)$ is simply based on the probabilities. We have:

$$
\operatorname{Prod}_{i}(j)=\prod_{k=1}^{j} \operatorname{sim}_{i \sigma_{i}(k)}
$$

where $\operatorname{Prod}_{i}(j)$ is the product of the first $\mathrm{j}$ ordered probabilities and $\sigma_{i}$ induces the order.

$$
N_{i j}=\sum_{k=1}^{j} r_{i \sigma_{i}(k)}
$$

where $N_{i j}$ is the sum of the normalized reliability associated with the $\mathrm{j}$ largest $\operatorname{sim} \times r$ products for the target case $C_{i}$.

We define $\mathrm{f}(\mathrm{x})$ as the weight generating function used to implement the desired soft likelihood function, then for $j=1 \ldots q$ we calculate the OWA weights associated with $C_{i}$ :

$$
w_{i j}=f\left(S_{i j}\right)-f\left(S_{i(j-i)}\right)
$$

Then the soft likelihood function for target case $C_{i}$ in the face of reliability is:

$$
S I M_{i, f}=\sum_{j=1}^{q} w_{i j} \operatorname{Prod}_{i}(j)
$$

If the reliability of $r_{i \sigma_{i}(k)}$ is $0, S_{i j}=S_{i(j-1)}$ and $w_{i j}=S_{i j}-S_{i(j-1)}=0$. If all the reliability are $r_{i j}=\frac{1}{q}, S_{i j}=\frac{j}{q}$ and $w_{i j}=f\left(\frac{j}{q}\right)-f\left(\frac{j-1}{q}\right)$. This is the same situation as not considering reliability. When $f(x)=x^{m}$ and $m=\frac{1-\alpha}{\alpha}$, we get $f(x)=x^{\frac{1-\alpha}{\alpha}}$ and the weight is

$$
w_{i j}=S_{i j}^{\frac{1-\alpha}{\alpha}}-S_{i(j-1)}^{\frac{1-\alpha}{\alpha}}
$$

Now we can improve our previous illustrative computations for the case where there are nonequal degrees of importance associated with the attribute. Next, we give an example to illustrate our case retrieval algorithm.

\section{Example 2:}

Let's have $q=6$ primary attributes. Local similarity with the 6 attributes between source case and target case is(the same as Example 1): $C=\left\{\operatorname{sim}_{i 1}=0.7, \operatorname{sim}_{i 2}=0.4, \operatorname{sim}_{i 3}=0.9, \operatorname{sim}_{i 4}=\right.$ $\left.1, \operatorname{sim}_{i 5}=0.5, \operatorname{sim}_{i 6}=0.8\right\}$. The associated non-normalized evidence reliability is: $R=\left\{R_{i 1}=\right.$ 
Table 4: Probability reliability

\begin{tabular}{cccc}
\hline $\mathrm{j}$ & Reliability $r_{i j}$ & Probability reliability $\operatorname{sim}_{i j} \times r_{i j}$ & Index order \\
\hline 1 & 0.244 & 0.171 & 1 \\
2 & 0.171 & 0.068 & 6 \\
3 & 0.097 & 0.088 & 5 \\
4 & 0.122 & 0.122 & 2 \\
5 & 0.220 & 0.110 & 4 \\
6 & 0.146 & 0.117 & 3 \\
\hline
\end{tabular}

$\left.1, R_{i 2}=0.7, R_{i 3}=0.4, R_{i 4}=0.5, R_{i 5}=0.9, R_{i 6}=0.6\right\}$. The normalized reliability is: $r_{i j}=$ $\frac{R_{i j}}{\sum_{k=1}^{q} R_{i k}}=\frac{R_{i j}}{4.1}$

We calculate the probability-reliability products as shown in Table 4.

Then the index function $\sigma_{i}(k)$ is: $\left\{\sigma_{i}(1)=1, \sigma_{i}(2)=4, \sigma_{i}(3)=6, \sigma_{i}(4)=5, \sigma_{i}(5)=3, \sigma_{i}(6)=\right.$ $2\}$.

We can calculate $\operatorname{Prod}_{i}(j)=\prod_{k=1}^{j} \operatorname{sim}_{i \sigma_{i}(k)}=\operatorname{Prod}_{i}(j-1) \operatorname{sim}_{i \sigma_{i}(j)}$ as shown in Table 5.

Table 5: Probability products

\begin{tabular}{ll}
\hline Ordered probability & $\operatorname{Prod}_{i}(j)$ \\
\hline $\operatorname{sim}_{i \sigma_{i}(1)}=\operatorname{sim}_{i 4}=0.7$ & $\operatorname{Prod}_{i}(1)=0.7$ \\
$\operatorname{sim}_{i \sigma_{i}(2)}=\operatorname{sim}_{i 1}=1$ & $\operatorname{Prod}_{i}(2)=0.7 \times 1=0.7$ \\
$\operatorname{sim}_{i \sigma_{i}(3)}=\operatorname{sim}_{i 6}=0.8$ & $\operatorname{Prod}_{i}(3)=0.7 \times 0.8=0.56$ \\
$\operatorname{sim}_{i \sigma_{i}(4)}=\operatorname{sim}_{i 5}=0.5$ & $\operatorname{Prod}_{i}(4)=0.56 \times 0.5=0.28$ \\
$\operatorname{sim}_{i \sigma_{i}(5)}=\operatorname{sim}_{i 3}=0.9$ & $\operatorname{Prod}_{i}(5)=0.28 \times 0.9=0.252$ \\
$\operatorname{sim}_{i \sigma_{i}(6)}=\operatorname{sim}_{i 2}=0.4$ & $\operatorname{Prod}_{i}(6)=0.252 \times 0.4=0.1008$ \\
\hline
\end{tabular}

We can use $N_{i j}=\sum_{k=1}^{j} r_{i \sigma_{i}(k)}=N_{i}(j-1)+r_{i \sigma_{i}(j)}$ calculate the normalized reliability based on the index $\sigma_{i}$ as shown in Table 6 .

For different $\alpha$, we can use $S I M_{i, \alpha}=\sum_{j=1}^{q} w_{i j} \operatorname{Prod}_{i}(j)$ to calculate the $S I M_{i, \alpha}$ with different reliability associated with the attribute and $w_{i j}=S_{i j}^{\frac{1-\alpha}{\alpha}}-S_{i(j-1)}^{\frac{1-\alpha}{\alpha}}$. Now we calculate some typical $S I M_{i, \alpha}$.

(1) $\alpha=0.8$ : This is a very optimistic attitudinal character. $m=\frac{1-\alpha}{\alpha}=0.25$. We can get Table 7. So $S I M_{i, \alpha}=0.617$ when $\alpha=0.8$. 
Table 6: Sum of normalized probabilities

\begin{tabular}{ll}
$r_{i \sigma_{i}(j)}$ & $N_{i j}$ \\
\hline$r_{i \sigma_{i}(1)}=0.244$ & $N_{i 1}=0.244$ \\
$r_{i \sigma_{i}(2)}=0.122$ & $N_{i 2}=0.366$ \\
$r_{i \sigma_{i}(3)}=0.146$ & $N_{i 3}=0.512$ \\
$r_{i \sigma_{i}(4)}=0.220$ & $N_{i 4}=0.732$ \\
$r_{i \sigma_{i}(5)}=0.097$ & $N_{i 5}=0.829$ \\
$r_{i \sigma_{i}(6)}=0.171$ & $N_{i 6}=1$ \\
\hline
\end{tabular}

Table 7: The numerical example of $\alpha=0.8$

\begin{tabular}{llllll}
\hline & $N_{i j}^{0.25}$ & $N_{i(j-1)}^{0.25}$ & $w_{i j}$ & $\operatorname{Prod}_{i}(j)$ & $w_{i j} \operatorname{Prod}_{i}(j)$ \\
\hline$j=1$ & 0.703 & 0 & 0.703 & 0.7 & 0.492 \\
$j=2$ & 0.778 & 0.703 & 0.075 & 0.7 & 0.052 \\
$j=3$ & 0.846 & 0.778 & 0.068 & 0.56 & 0.038 \\
$j=4$ & 0.925 & 0.846 & 0.079 & 0.28 & 0.022 \\
$j=5$ & 0.954 & 0.925 & 0.029 & 0.252 & 0.0074 \\
$j=6$ & 1 & 0.954 & 0.046 & 0.1008 & 0.0046 \\
& & & $\sum_{j} w_{i j}=1$ & & $\sum_{j} w_{i j} \operatorname{Prod}_{i}(j)=0.617$ \\
\hline
\end{tabular}


(2) $\alpha=0.5$ : This is a very neutral attitudinal character. $m=\frac{1-\alpha}{\alpha}=1$. We can get Table 8 . So $S I M_{i, \alpha}=0.441$ when $\alpha=0.5$.

Table 8: The numerical example of $\alpha=0.5$

\begin{tabular}{llllll}
\hline & $N_{i j}$ & $N_{i(j-1)}$ & $w_{i j}$ & $\operatorname{Prod}_{i}(j)$ & $w_{i j} \operatorname{Prod}_{i}(j)$ \\
\hline$j=1$ & 0.244 & 0 & 0.244 & 0.7 & 0.171 \\
$j=2$ & 0.366 & 0.244 & 0.122 & 0.7 & 0.085 \\
$j=3$ & 0.512 & 0.366 & 0.146 & 0.56 & 0.082 \\
$j=4$ & 0.732 & 0.512 & 0.220 & 0.28 & 0.062 \\
$j=5$ & 0.829 & 0.732 & 0.097 & 0.252 & 0.024 \\
$j=6$ & 1 & 0.829 & 0.171 & 0.1008 & 0.017 \\
& & & $\sum_{j} w_{i j}=1$ & & $\sum_{j} w_{i j} \operatorname{Prod}_{i}(j)=0.441$ \\
\hline
\end{tabular}

(3) $\alpha=0.2$ : This is a very pessimistic attitudinal character. $m=\frac{1-\alpha}{\alpha}=4$. We can get Table 9. So $S I M_{i, \alpha}=0.202$ when $\alpha=0.2$.

Table 9: The numerical example of $\alpha=0.2$

\begin{tabular}{llllll}
\hline & $N_{i j}^{4}$ & $N_{i(j-1)}^{4}$ & $w_{i j}$ & $\operatorname{Prod}_{i}(j)$ & $w_{i j} \operatorname{Prod}_{i}(j)$ \\
\hline$j=1$ & 0.0035 & 0 & 0.0035 & 0.7 & 0.0025 \\
$j=2$ & 0.018 & 0.0035 & 0.0144 & 0.7 & 0.0101 \\
$j=3$ & 0.069 & 0.018 & 0.051 & 0.56 & 0.028 \\
$j=4$ & 0.287 & 0.069 & 0.218 & 0.28 & 0.061 \\
$j=5$ & 0.472 & 0.287 & 0.185 & 0.252 & 0.047 \\
$j=6$ & 1 & 0.472 & 0.528 & 0.1008 & 0.053 \\
& & & $\sum_{j} w_{i j}=1$ & & $\sum_{j} w_{i j} \operatorname{Prod}_{i}(j)=0.202$ \\
\hline
\end{tabular}

In the above we clearly see the increasing nature of the soft likelihood value from Table 10 as the degree of optimism $\alpha$ increases.

Finally let us try to develop a more formal understanding of the mechanics underlying this approach for calculating the soft-likelihood associated with a decision maker. Again using the index function for the probability-reliability product we have $\operatorname{Prod}(j)=\prod_{k=1}^{j} \operatorname{sim}_{\sigma_{(k)}}$ and $N_{j}=$ $\sum_{k=1}^{j} r_{\sigma(k)}$. 
Table 10: As optimism $\alpha$ increases.

\begin{tabular}{cc} 
Optimism $\alpha$ & Soft likelihood value \\
\hline 0.2 & 0.202 \\
0.5 & 0.441 \\
0.8 & 0.617 \\
\hline
\end{tabular}

We then calculate the soft likelihood value $S I M_{f}$ using an OWA aggregation based on the weight generating function $\mathrm{f}:[0,1] \longrightarrow[1,0]$. Here then $S I M_{f}=\sum_{j=1}^{q} w_{j} \operatorname{Prod}(j)$, where $w_{j}=$ $f\left(S_{j}\right)-f\left(S_{(j-i)}\right)$.

We note the characterizing parameter is $\alpha=\int_{0}^{1} f(y) d y$. $\alpha$ ranges from 1 , most optimistic; to $\alpha=0$, most pessimistic. Here to capture the more general behavior, we will use $\alpha=0.5$, a neutral case.

The retrieval strategy we proposed is to combine the case retrieval algorithm based on soft likelihood functions developed above with KNN, replacing the traditional KNN strategy combined with the ordinary mean algorithm or the weight average method, so as to improve the accuracy of case retrieval in CBR.

\section{Experimental verification}

In this section, we describe and simulate the algorithm proposed in this paper to evaluate the effectiveness of the proposed case retrieval method. We selected 10 classification data sets from UCI resource base for classification experiment. The abbreviations of names, number of samples, number of attributes, number of categories and other information of each data set are shown in Table 11. Detailed descriptions of each dataset are omitted here.

In this paper, our main contribution is to develop a case retrieval algorithm based on soft likelihood functions, and apply the proposed CBR-SLFs method to KNN to obtain a new CBR retrieval strategy. In order to make a fair and detailed comparison, it is necessary to compare its performance with traditional retrieval strategies. At present, the retrieval strategy based on KNN generally uses average-based method when calculating the similarity between the target case and the source case in the case base.

The experimental process is as follows. Firstly, the data set is divided into training set and test 
Table 11: The general information of the used data sets.

\begin{tabular}{ccccc} 
Data set & Instance & Class & Attribute & Area \\
\hline Iris & 150 & 3 & 4 & Life \\
Balance & 625 & 3 & 4 & Social \\
Survival & 306 & 2 & 3 & Life \\
Endgame & 958 & 2 & 9 & Game \\
Blood & 748 & 2 & 4 & Business \\
Banknote & 1372 & 2 & 4 & Computer \\
Breast & 116 & 2 & 9 & Life \\
Vertebral & 310 & 2 & 6 & N/A \\
User & 403 & 4 & 5 & Computer \\
Wholesale & 440 & 2 & 7 & Business \\
\hline
\end{tabular}

set by using the 10-fold cross validation method. The training set is used as the historical case base, and each case in the test set is used as the target case. Based on the case base, different retrieval strategies are used to find solutions for each target case. If the obtained solution is consistent with the corresponding solution of the test set, the retrieval strategy is considered to be effective. The effectiveness of the retrieval strategy is represented by the ratio of the number of cases with effective solutions to the number of elements in the test set. For all data sets, the process is repeated 100 times and a simple average is reported.

In order to verify the effect of case retrieval strategy of CBR-SLFs proposed in this paper on CBR classification accuracy, the following 5 case retrieval algorithms were used for comparative experiments:

(1)The KNN retrieval strategy based on mean operator is used to investigate the performance of case retrieval, denoted as KNN-Mean;

(2)The KNN retrieval strategy based on trim mean operator is used to investigate the performance of case retrieval, denoted as KNN-Trim;

(3)The KNN retrieval strategy based on weighted average operator is used to investigate the performance of case retrieval, denoted as KNN-Weight;

(4)The KNN retrieval strategy based on SLFs operator proposed in this paper is used to investigate the performance of case retrieval, denoted as KNN-SLF; 
(5)The KNN retrieval strategy based on SLFs operator considering attribute reliability proposed in this paper is used to investigate the performance of case retrieval, denoted as KNN-RESLF.

Note that since the data set used in the experiment does not provide the degree of reliability of the feature, we use a random method to generate the degree of reliability of the attribute.

For the KNN, we study the case of $\mathrm{k}$ values between 5 and 20. As can be seen from Fig. 2, different $\mathrm{K}$ values have little influence on the efficiency of the retrieval strategy. The efficiency of the retrieval strategy is basically flat but fluctuates slightly, indicating that the retrieval strategy is not very sensitive to $\mathrm{K}$. In the comparison test, take $k=11$.
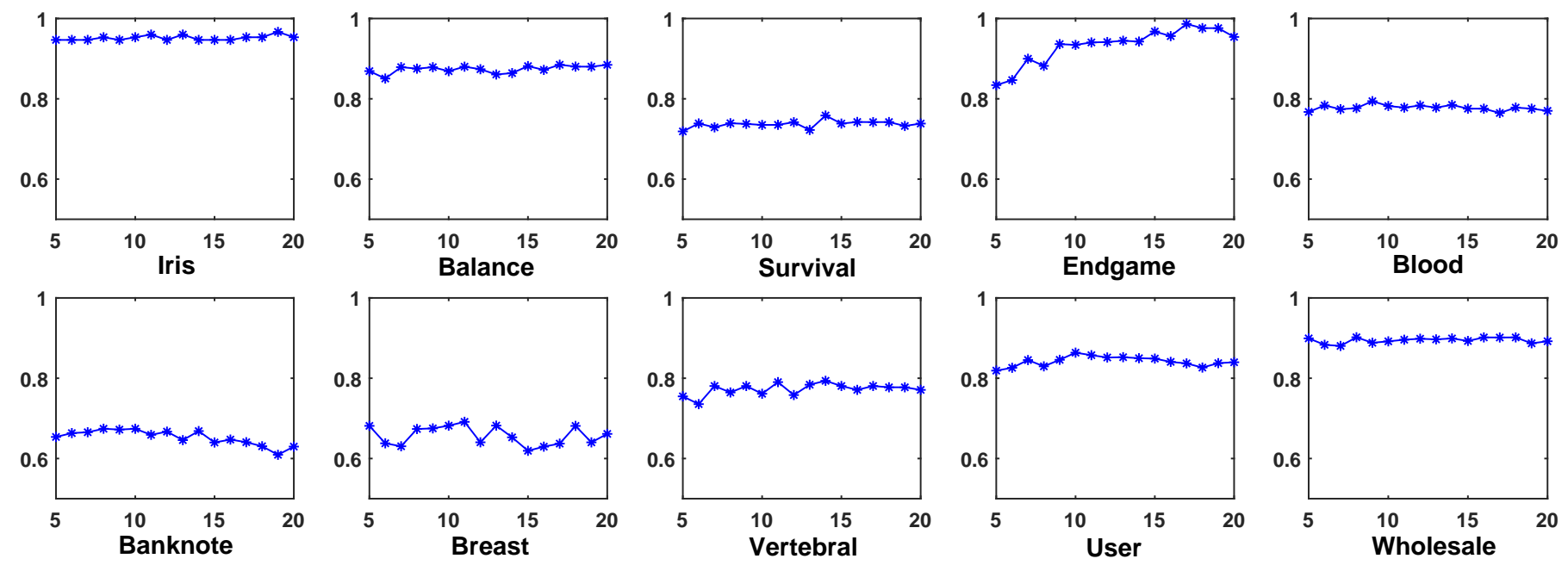

Figure 2: The performance of the retrieval strategy with KNN-RESLF algorithm under different K.

The soft likelihood functions involve the DMs' attitude parameter $\alpha$. Fig. 3 shows the influence of the value of $\alpha$ from $0 \ldots 1$, that is, the DMs' attitude from negative to positive, on the correctness of the retrieval strategy. It can be seen that the selection of parameters and different data set types will have impact on the retrieval effect, and the value of $\alpha$ needs to be determined according to the characteristics of the actual decision maker and the field in which the case is located. In the comparison test, take the DMs' attitude is neutral, i.e., $\alpha=0.5$.

We obtained the accuracy of different retrieval strategies in each data set, as shown in Table 12. In order to compare the performance of different retrieval strategies more clearly, we average the accuracy of each retrieval strategy in all data sets to represent the performance of the 5 retrieval 

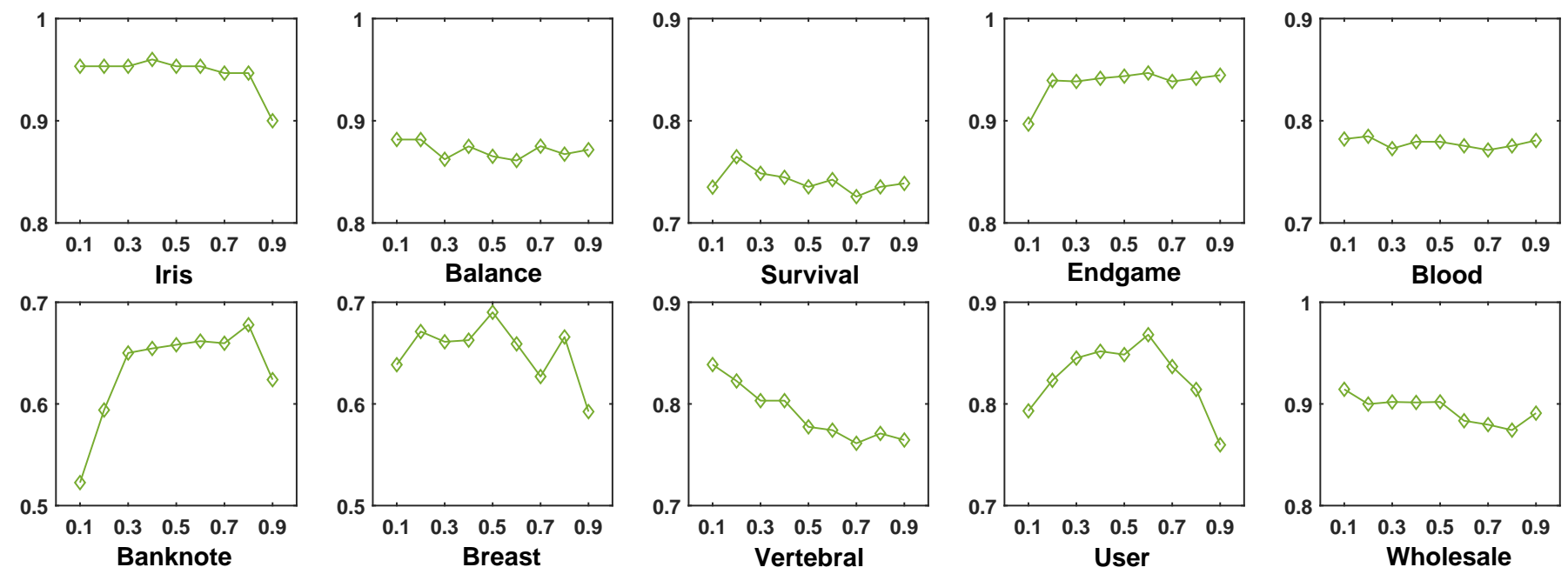

Figure 3: The performance of the retrieval strategy with KNN-RESLF algorithm under different $\alpha$.

Table 12: The performance of case-based reasoning with different retrieval strategy.

\begin{tabular}{cccccc} 
Data set & Knn-Mean & Knn-Trim & KNN-Weight & Knn-SLF & Knn-RESLF \\
\hline Iris & 0.9545 & 0.9555 & 0.9541 & 0.9548 & 0.9526 \\
Balance & 0.8104 & 0.7625 & 0.8313 & 0.8716 & 0.8725 \\
Survival & 0.7273 & 0.7264 & 0.7334 & 0.7417 & 0.7429 \\
Endgame & 0.8907 & 0.8584 & 0.9015 & 0.9373 & 0.9417 \\
Blood & 0.7579 & 0.7589 & 0.7579 & 0.7753 & 0.7757 \\
Banknote & 0.5698 & 0.6024 & 0.5728 & 0.6505 & 0.6507 \\
Breast & 0.6634 & 0.6760 & 0.6798 & 0.6781 & 0.6726 \\
Vertebral & 0.8126 & 0.7836 & 0.8086 & 0.8242 & 0.8284 \\
User & 0.8258 & 0.8282 & 0.8234 & 0.8457 & 0.8462 \\
Wholesale & 0.8634 & 0.8702 & 0.9037 & 0.8967 & 0.8934 \\
\hline Average & 0.7876 & 0.7822 & 0.7967 & 0.8176 & 0.8177 \\
\hline
\end{tabular}


strategies. As can be seen from Table 12:

(1)The retrieval strategy trim mean-based algorithm is the worst in almost all data sets;

(2)The retrieval strategies of KNN-SLF and KNN-RESLF are better than other retrieval strategies;

(3)The ranking of average retrieval efficiency based on all data sets can be obtained by various retrieval strategies: $K N N-R E S L F \approx K N N-S L F>K N N-$ Weight $>K N N-$ Mean $>K N N-$ Trim.

The above analysis can illustrate the advantages of the retrieval strategy based on SLFs proposed improved to improve the adaptability and reliability of the method. Secondly, this study only covers 
a limited number of feature types. Considering the various data types that may exist in the actual CBR process, we will enrich the feature types more comprehensively in the following research. Next, the attributes of a case are not completely unrelated. We can combine the characteristics of specific research problems to study the interaction between attributes. And in the future, CBR can be applied to solve complex problems in practice, such as disease diagnosis and image recognition and so on.

\section{Acknowledgments}

390 This research was funded by the grants from the National Natural Science Foundation of China (\#72034001, \#71974044, \#91646105).

\section{References}

[1] R. C. Schank, Dynamic memory: A theory of reminding and learning in computers and people, Cambridge University Press, 1983.

[2] J. L. Kolodner, R. L. Simpson, The mediator: Analysis of an early case-based problem solver, Cognitive Science 13 (4) (1989) 507-549.

[3] M. Navarro, S. Heras, V. Julián, V. Botti, Incorporating temporal-bounded cbr techniques in real-time agents, Expert Systems with Applications 38 (3) (2011) 2783-2796.

[4] G. Müller, R. Bergmann, Learning and applying adaptation operators in process-oriented casebased reasoning, in: International Conference on Case-Based Reasoning, Springer, 2015, pp. $259-274$.

[5] T. P. D. Homem, P. E. Santos, A. H. R. Costa, R. A. da Costa Bianchi, R. L. de Mantaras, Qualitative case-based reasoning and learning, Artificial Intelligence 283 (2020) 103258.

[6] D. V.-K. Le, Z. Chen, Y. W. Wong, D. Isa, A complete online-svm pipeline for case-based reasoning system: a study on pipe defect detection system, Soft Computing 24 (2020) 1691716933. 
[7] E. Armengol, A. Palaudaries, E. Plaza, Individual prognosis of diabetes long-term risks: A cbr approach, Methods of Information in Medicine-Methodik der Information in der Medizin 40 (1) (2001) 46-51.

[8] R. Schmidt, L. Gierl, A prognostic model for temporal courses that combines temporal abstraction and case-based reasoning, International journal of medical informatics 74 (2-4) (2005) $307-315$.

[9] C. Jian, T. Zhe, L. Zhenxing, A review and analysis of case-based reasoning research, in: 2015 International Conference on Intelligent Transportation, Big Data and Smart City, IEEE, 2015, pp. 51-55.

[10] W. Liu, R. Tan, G. Cao, F. Yu, H. Li, Creative design through knowledge clustering and case-based reasoning, Engineering with Computers (2019) 1-15.

[11] A. Holt, I. Bichindaritz, R. Schmidt, P. Perner, Medical applications in case-based reasoning, Knowledge Engineering Review 20 (3) (2005) 289-292.

[12] V. C. Georgopoulos, C. D. Stylios, Complementary case-based reasoning and competitive fuzzy cognitive maps for advanced medical decisions, Soft Computing 12 (2) (2008) 191-199.

[13] S. Begum, M. U. Ahmed, P. Funk, N. Xiong, M. Folke, Case-based reasoning systems in the health sciences: a survey of recent trends and developments, IEEE Transactions on Systems, Man, and Cybernetics, Part C (Applications and Reviews) 41 (4) (2010) 421-434.

[14] J. Ramos-González, D. López-Sánchez, J. A. Castellanos-Garzón, J. F. de Paz, J. M. Corchado, A cbr framework with gradient boosting based feature selection for lung cancer subtype classification, Computers in biology and medicine 86 (2017) 98-106.

[15] F. Torrent-Fontbona, J. Massana, B. López, Case-base maintenance of a personalised and adaptive cbr bolus insulin recommender system for type 1 diabetes, Expert Systems with Applications 121 (2019) 338-346.

[16] T. Pinto, R. Faia, M. Navarro-Caceres, G. Santos, J. M. Corchado, Z. Vale, Multi-agentbased cbr recommender system for intelligent energy management in buildings, IEEE Systems Journal 13 (1) (2018) 1084-1095. 
[17] Z. Jiang, Y. Jiang, Y. Wang, H. Zhang, H. Cao, G. Tian, A hybrid approach of rough set and case-based reasoning to remanufacturing process planning, Journal of Intelligent Manufacturing 30 (1) (2019) 19-32.

[18] C. Liang, D. Gu, I. Bichindaritz, X. Li, C. Zuo, W. Cheng, Integrating gray system theory and logistic regression into case-based reasoning for safety assessment of thermal power plants, Expert Systems with Applications 39 (5) (2012) 5154-5167.

[19] T. Hong, C. Koo, D. Kim, M. Lee, J. Kim, An estimation methodology for the dynamic operational rating of a new residential building using the advanced case-based reasoning and stochastic approaches, Applied Energy 150 (2015) 308-322.

[20] N. Kwon, K. Song, Y. Ahn, M. Park, Y. Jang, Maintenance cost prediction for aging residential buildings based on case-based reasoning and genetic algorithm, Journal of Building Engineering 28 (2020) 101006.

[21] L. Xu, C. Huang, J. Niu, C. Li, J. Wang, H. Liu, X. Wang, An improved case-based reasoning method and its application to predict machining performance, Soft Computing 25 (7) (2021) $5683-5697$.

[22] L. Catalá, V. Julián, J.-A. Gil-Gómez, A cbr-based game recommender for rehabilitation videogames in social networks, in: International Conference on Intelligent Data Engineering and Automated Learning, Springer, 2014, pp. 370-377.

[23] G. Alshammari, J. L. Jorro-Aragoneses, S. Kapetanakis, M. Petridis, J. A. Recio-García, B. Díaz-Agudo, A hybrid cbr approach for the long tail problem in recommender systems, in: International Conference on Case-Based Reasoning, Springer, 2017, pp. 35-45.

[24] A. González-Briones, J. Prieto, F. De La Prieta, E. Herrera-Viedma, J. M. Corchado, Energy optimization using a case-based reasoning strategy, Sensors 18 (3) (2018) 865.

[25] M. W. Floyd, M. Drinkwater, D. W. Aha, Trust-guided behavior adaptation using case-based reasoning, Tech. rep., Naval Research Laboratory Washington United States (2015).

[26] F. Le Ber, J. Lieber, M. Benoit, Case-based reasoning for forecasting the allocation of perennial biomass crops, ERCIM News (113) (2018) 34-35. 
[27] A. Aamodt, E. Plaza, Case-based reasoning: Foundational issues, methodological variations, and system approaches, AI communications 7 (1) (1994) 39-59.

[28] R. Bergmann, J. Kolodner, E. Plaza, Representation in case-based reasoning, Knowledge Engineering Review 20 (3) (2005) 209-214.

[29] R. L. De Mantaras, D. McSherry, D. Bridge, D. Leake, B. Smyth, S. Craw, B. Faltings, M. L. Maher, M. T COX, K. Forbus, et al., Retrieval, reuse, revision and retention in case-based reasoning, The Knowledge Engineering Review 20 (3) (2005) 215-240.

[30] Y.-B. Kang, S. Krishnaswamy, A. Zaslavsky, A retrieval strategy for case-based reasoning using similarity and association knowledge, IEEE transactions on cybernetics 44 (4) (2013) 473-487.

[31] S. Petrovic, G. Khussainova, R. Jagannathan, Knowledge-light adaptation approaches in casebased reasoning for radiotherapy treatment planning, Artificial intelligence in medicine 68 (2016) 17-28.

[32] V. S. Rallabandi, S. Sett, Knowledge-based image retrieval system, Knowledge-Based Systems 21 (2) (2008) 89-100.

[33] L. Chen, Z.-H. Cheng, H.-Y. Zeng, Study on case retrieval of case-based rcm analysis system, Computer Engineering and Design 33 (2) (2012) 581-585.

[34] T. Cover, P. Hart, Nearest neighbor pattern classification, IEEE transactions on information theory 13 (1) (1967) 21-27.

[35] X. Guo, J. Yuan, Y. Li, Feature space k nearest neighbor based batch process monitoring, Acta Autom. Sin 40 (1) (2014) 135-142.

[36] D. Greene, J. Freyne, B. Smyth, P. Cunningham, An analysis of current trends in cbr research using multi-view clustering, AI Magazine 31 (2) (2010) 45-45.

[37] R. Schmidt, S. Montani, R. Bellazzi, L. Portinale, L. Gierl, Cased-based reasoning for medical knowledge-based systems, International Journal of Medical Informatics 64 (2-3) (2001) 355367.

[38] H. Li, J. Sun, B.-L. Sun, Financial distress prediction based on or-cbr in the principle of k-nearest neighbors, Expert Systems with Applications 36 (1) (2009) 643-659. 
[39] S.-W. Lin, S.-C. Chen, Parameter tuning, feature selection and weight assignment of features for case-based reasoning by artificial immune system, Applied Soft Computing 11 (8) (2011) $5042-5052$.

[40] R. R. Yager, P. Elmore, F. Petry, Soft likelihood functions in combining evidence, Information Fusion 36 (2017) 185-190.

[41] Y. Tian, X. Mi, L. Liu, B. Kang, A new soft likelihood function based on d numbers in handling uncertain information, International Journal of Fuzzy Systems 22 (7) (2020) 2333-2349.

[42] R. R. Yager, On ordered weighted averaging aggregation operators in multicriteria decisionmaking, IEEE Transactions on systems, Man, and Cybernetics 18 (1) (1988) 183-190.

[43] R. R. Yager, Quantifier guided aggregation using owa operators, International Journal of Intelligent Systems 11 (1) (1996) 49-73.

[44] P. Cunningham, A taxonomy of similarity mechanisms for case-based reasoning, IEEE Transactions on Knowledge and Data Engineering 21 (11) (2008) 1532-1543.

[45] S. El-Sappagh, M. Elmogy, F. Ali, K.-S. Kwak, A case-base fuzzification process: diabetes diagnosis case study, Soft Computing 23 (14) (2019) 5815-5834.

[46] G. Yu, D. Li, Y. Ye, J. Qiu, Heterogeneous multi-attribute variable weight decision-making method considering regret avoidance, Comput. Integr. Manuf. Syst.(Chin.) 23 (2017) 154-161.

[47] P. Yahong, G. Xiuli, A hybrid multiple attributes group decision making method based on vikor, Machine Design and Research 34 (1) (2018) 177-182.

[48] S.-P. Wan, J. Xu, J.-Y. Dong, Aggregating decision information into interval-valued intuitionistic fuzzy numbers for heterogeneous multi-attribute group decision making, Knowledge-Based Systems 113 (2016) 155-170.

[49] H. Nikpour, A. Aamodt, Fault diagnosis under uncertain situations within a bayesian knowledge-intensive cbr system, Progress in Artificial Intelligence (2021) 1-14.

[50] L. Fei, Y. Feng, L. Liu, On pythagorean fuzzy decision making using soft likelihood functions, International Journal of Intelligent Systems 34 (12) (2019) 3317-3335. 
[51] R.-P. Tan, W.-D. Zhang, S.-Q. Chen, L.-H. Yang, Emergency decision-making method based on case-based reasoning in heterogeneous information environment, Control and Decision 35 (2020) 1966-1976.

[52] H. A. Simon, A behavioral model of rational choice, The quarterly journal of economics 69 (1) (1955) 99-118.

[53] X. Mi, Y. Tian, B. Kang, A hybrid multi-criteria decision making approach for assessing healthcare waste management technologies based on soft likelihood function and d-numbers, Applied Intelligence (2021) 1-20.

[54] L. Fei, Y. Feng, A novel retrieval strategy for case-based reasoning based on attitudinal choquet integral, Engineering Applications of Artificial Intelligence 94 (2020) 103791.

[55] Y. Li, S. C. Shiu, S. K. Pal, Combining feature reduction and case selection in building cbr classifiers, iEEE Transactions on knowledge and Data Engineering 18 (3) (2006) 415-429.

[56] D. McSherry, Conversational case-based reasoning in medical decision making, Artificial Intelligence in Medicine 52 (2) (2011) 59-66.

[57] L. Fei, Y. Feng, L. Liu, W. Mao, On intuitionistic fuzzy decision-making using soft likelihood functions, International Journal of Intelligent Systems 34 (9) (2019) 2225-2242. 


\section{Supplementary Files}

This is a list of supplementary files associated with this preprint. Click to download.

- declaration.docx 